(C) 2004 Springer. This is the author version of an article published in a Springer journal. The original publication is available at springerlink.com with DOI: 10.1007/s00041-004-3072-z

\title{
ON A PROBLEM OF GRÖCHENIG ABOUT NONLINEAR APPROXIMATION WITH LOCALIZED FRAMES
}

\author{
RÉMI GRIBONVAL ${ }^{\star}$ AND MORTEN NIELSEN $^{\star \ddagger}$
}

\begin{abstract}
We prove that the exponential localization of a frame with respect to an orthonormal basis in a Hilbert space is not sufficient to get a Bernstein inequality. In other words, the fact that a function belongs to an approximation space of the frame cannot be characterized in terms of the sparseness of its frame coefficients.
\end{abstract}

\section{INTRODUCTION}

In a Hilbert space $\mathcal{H}$ of infinite dimension, a dictionary is any family $\mathcal{D}=\left\{g_{k}\right\}$ of quasinormalized vectors $\left(0<c \leq\left\|g_{k}\right\| \leq C<\infty\right.$ for all $\left.k\right)$ with dense span. Given a dictionary in $\mathcal{H}$, one can consider the problem of nonlinear approximation of any vector $f \in \mathcal{H}$ using $m$-term expansions from $\mathcal{D}$. Considering the (nonlinear) manifold

$$
\Sigma_{m}(\mathcal{D}):=\left\{f=\sum_{k \in \Lambda} c_{k} g_{k}, c_{k} \in \mathbb{C},|\Lambda| \leq m\right\}
$$

of $m$-term expansions from $\mathcal{D}$, the error of best $m$-term approximation of $f$ from $\mathcal{D}$ is

$$
\sigma_{m}(f, \mathcal{D}):=\operatorname{dist}\left(f, \Sigma_{m}(\mathcal{D})\right)=\inf _{h \in \Sigma_{m}(\mathcal{D})}\|f-h\| .
$$

One of the central problems of (nonlinear) approximation theory is to characterize the set of vectors $f \in \mathcal{H}$ which can be approximated at a certain rate using $m$-term expansions. With this aim, one defines the approximation classes

$$
\mathcal{A}_{q}^{\alpha}(\mathcal{D}):=\left\{f \in \mathcal{H},|f|_{\mathcal{A}_{q}^{\alpha}(\mathcal{D})}:=\left(\sum_{m \geq 1}\left[m^{\alpha} \sigma_{m}(f, \mathcal{D})\right]^{q} \frac{1}{m}\right)^{1 / q}<\infty\right\}
$$

for $0<\alpha<\infty$ and $q \in(0, \infty]$. A suitably modified definition is used for $q=\infty$. Standard results from approximation theory [3, Chapter 7] show that $\mathcal{A}_{q}^{\alpha}(\mathcal{D})$ is a (quasi)Banach space when equipped with the (quasi)norm $\|\cdot\|_{\mathcal{A}_{q}^{\alpha}(\mathcal{D})}:=\|\cdot\|+|\cdot|_{\mathcal{A}_{q}^{\alpha}(\mathcal{D})}$. When $\mathcal{D}$ is an orthonormal basis of $\mathcal{H}$, the results of Stechkin [10], DeVore and Temlyakov [4] show that the approximation space can be identified (with equivalent norms) with the sparseness class

$$
\mathcal{H}_{\tau, q}(\mathcal{D}):=\left\{f \in \mathcal{H},\|f\|_{\mathcal{H}_{\tau, q}(\mathcal{D})}:=\left\|\left\{\left\langle f, g_{k}\right\rangle\right\}_{k}\right\|_{\ell_{\tau, q}}<\infty\right\}
$$

where $\ell_{\tau, q}$ is a rearrangement invariant Lorentz space [1] and $\alpha=1 / \tau-1 / 2$. A similar characterization is valid for Riesz bases, and the result has been generalized by the authors [5] to nice Schauder bases in more general Banach spaces, where the relation $\alpha=1 / \tau-1 / 2$ has to be replaced with $\alpha=1 / \tau-1 / p$ for some appropriate $p \in(1, \infty)$.

When $\mathcal{D}$ is a redundant dictionary in the Hilbert space $\mathcal{H}$, DeVore and Temlyakov [4] suggested to replace $\mathcal{H}_{\tau, q}$ with a larger class $\mathcal{K}_{\tau, q} \supset \mathcal{H}_{\tau, q}$ which is defined in terms of the sparseness of the

Key words and phrases. Redundant dictionary, frame, localized frame, nonlinear approximation, Jackson inequality, Bernstein inequality.

${ }^{\star}$ Partially supported by the Danish Technical Science Foundation, Grant no. 9701481. ${ }^{\triangle}$ Partially supported by European Union's Human Potential Programme, under contract HPRN-CT-2002-00285 (HASSIP). ${ }^{\ddagger}$ Partially supported by the Danish SNF-PDE network. 
(c) 2004 Springer. This is the author version of an article published in a Springer journal. The original publication is available at springerlink.com with DOI: 10.1007/s00041-004-3072-z

"sparsest synthesis coefficients" rather than that of the analysis coefficients $\left\{\left\langle f, g_{k}\right\rangle\right\}$. However, it is not known under what conditions $\mathcal{A}_{q}^{\alpha}(\mathcal{D})=\mathcal{K}_{\tau, q}(\mathcal{D})$.

On the one hand, it is now well understood [6] that the continuous embedding $\mathcal{K}_{\tau, q}(\mathcal{D}) \hookrightarrow$ $\mathcal{A}_{q}^{\alpha}(\mathcal{D})$ (which is called a direct estimate, or a Jackson embedding) for all $0<\alpha=1 / \tau-1 / 2<\infty$ is almost equivalent to the fact that $\mathcal{D}$ is $\ell_{2,1}$-Hilbertian, i.e., that the synthesis operator $D$ : $\left\{c_{k}\right\} \mapsto \sum_{k} c_{k} g_{k}$ is bounded from $\ell_{2,1}$ to $\mathcal{H}$. On the other hand, it seems quite difficult to find general conditions that would be sufficient to get the converse embedding $\mathcal{A}_{q}^{\alpha}(\mathcal{D}) \hookrightarrow \mathcal{K}_{\tau, q}(\mathcal{D})$, $\alpha=1 / \tau-1 / 2$. From standard results on the interplay between approximation theory and interpolation theory [3, Chapter 7], such an embedding is almost equivalent to the so-called Bernstein inequality

$$
|h|_{\mathcal{K}_{\tau, q}(\mathcal{D})} \leq C m^{\alpha}\|h\|, \forall m \geq 1, \forall h \in \Sigma_{m}(\mathcal{D})
$$

More precisely, the converse embedding implies (1.1), and (1.1) implies that, for all $0<\beta<\alpha$ and $s \in(0, \infty]$, we have the embedding $\mathcal{A}_{s}^{\beta}(\mathcal{D}) \hookrightarrow\left(\mathcal{H}, \mathcal{K}_{\tau, q}(\mathcal{D})\right)_{\beta / \alpha, s}$, where $(X, Y)_{\theta, s}$ is a real interpolation space between $X$ and $Y[1]$.

A widely studied family of dictionaries which obviously satisfy the $\ell_{2,1}$-Hilbertian condition is the family of frames [2]: a countable family $\left\{g_{k}\right\}$ in a separable Hilbert space $\mathcal{H}$ is called a frame for $\mathcal{H}$ if there exists two constants $0<A \leq B<\infty$ such that for all $f \in \mathcal{H}$

$$
A\|f\|^{2} \leq \sum_{k}\left|\left\langle f, g_{k}\right\rangle\right|^{2} \leq B\|f\|^{2} .
$$

Being a frame is equivalent to the fact that the synthesis operator $D:\left\{c_{k}\right\} \mapsto \sum_{k} c_{k} g_{k}$ is onto and bounded from $\ell_{2}$ to $\mathcal{H}$. Thus, quasi-normalized frames are dictionaries that satisfy the direct estimate $\mathcal{K}_{\tau, q}(\mathcal{D}) \hookrightarrow \mathcal{A}_{q}^{\alpha}(\mathcal{D}), 0<\alpha=1 / \tau-1 / 2<\infty$, and it is natural to wonder what additional assumptions on a quasi-normalized frame $\mathcal{D}$ will also ensure that the Bernstein inequality is satisfied.

Recently, Gröchenig introduced the notion of localized frames [7] and suggested [8] that localization might be a sufficient condition to have a Bernstein inequality. The purpose of this paper is to disprove this suggestion. Let $\|\cdot\|_{\mathbb{R}^{d}}$ be any of the equivalent norms on $\mathbb{R}^{d}$ and let $K, \mathcal{N} \subset \mathbb{R}^{d}$ be two separated countable index sets, in the sense that $\inf _{k, \ell \in K, k \neq \ell}\|k-\ell\|_{\mathbb{R}^{d}}>0$ and likewise for $\mathcal{N}$. Let $\mathcal{B}=\left\{e_{n}\right\}_{n \in \mathcal{N}}$ be a Riesz basis for $\mathcal{H}$ and $\tilde{\mathcal{B}}=\left\{\tilde{e}_{n}\right\}_{n \in \mathcal{N}}$ its dual basis. A frame $\mathcal{D}=\left\{g_{k}\right\}_{k \in K}$ is polynomially localized w.r.t $\mathcal{B}$ with decay $s>0$ if there exists a constant $C<\infty$ such that

$$
\max \left(\left|\left\langle g_{k}, e_{n}\right\rangle\right|,\left|\left\langle g_{k}, \tilde{e}_{n}\right\rangle\right|\right) \leq C\left(1+\|k-n\|_{\mathbb{R}^{d}}\right)^{-s} .
$$

It is exponentially localized w.r.t. $\mathcal{B}$ if for some $\lambda>0$ and $C<\infty$

$$
\max \left(\left|\left\langle g_{k}, e_{n}\right\rangle\right|,\left|\left\langle g_{k}, \tilde{e}_{n}\right\rangle\right|\right) \leq C \exp \left(-\lambda\|k-n\|_{\mathbb{R}^{d}}\right) .
$$

An important property of localized frames is the equality with equivalent norms

$$
\mathcal{H}_{\tau, q}(\mathcal{B})=\mathcal{H}_{\tau, q}(\tilde{\mathcal{B}})=\mathcal{H}_{\tau, q}(\mathcal{D})=\mathcal{K}_{\tau, q}(\mathcal{D}), \quad q \in(0, \infty]
$$

which is valid for $d / s<\tau<2$ when $\mathcal{D}$ is polynomially localized with decay $s>d$, and for $0<\tau<2$ when it is exponentially localized. Localized frames include many frames that are familiar to harmonic analysts. In particular, "nice" Gabor frames in $\mathcal{H}=L_{2}\left(\mathbb{R}^{d}\right)$ are localized w.r.t Wilson bases, but no Bernstein inequality for them has been proved so far. Since the localization of a frame is a priori much easier to check than a Bernstein inequality, proving that every localized frame satisfies a Bernstein inequality would be a major step forward in nonlinear approximation theory. Unfortunately such a general result does not hold: in this paper we build a quasi-normalized frame $\mathcal{D}$ which is exponentially localized w.r.t. some orthonormal basis $\mathcal{B}$, yet it fails to satisfy a Bernstein inequality. 
(C) 2004 Springer. This is the author version of an article published in a Springer journal. The original publication is available at springerlink.com with DOI: 10.1007/s00041-004-3072-z

\section{A SPECIFIC LOCALIZED FRAME With NO BERnstein INEQUALity}

Let $\mathcal{B}=\left\{e_{k}\right\}_{k \geq 1}$ be an orthonormal basis for a Hilbert space $\mathcal{H}$. The main step is to construct a second Riesz basis $\mathcal{B}^{\prime}=\left\{f_{k}\right\}_{k \geq 1}$ by perturbing $\mathcal{B}$. For $k \geq 1$, we will define $f_{k}:=e_{k}-\varepsilon_{k} h_{k}$ with $\varepsilon_{k} \in \mathbb{R}$ and the perturbation $\varepsilon_{k} h_{k}$ "small enough" to ensure that $\mathcal{B}^{\prime}$ is a Riesz basis exponentially localized w.r.t. $\mathcal{B}$. We will choose $h_{k}$ such that, for all $0<\tau<2$ and $q \in(0, \infty]$, $\sup _{k}\left|h_{k}\right|_{\mathcal{H}_{\tau, q}(\mathcal{B})} /\left\|h_{k}\right\|=\infty$. Since $h_{k}=\left(e_{k}-f_{k}\right) / \varepsilon_{k} \in \Sigma_{2}\left(\mathcal{B} \cup \mathcal{B}^{\prime}\right)$ and $\mathcal{D}=\mathcal{B} \cup \mathcal{B}^{\prime}$ will be exponentially localized w.r.t. $\mathcal{B}$ too, the result will follow.

We define $h_{k}:=\sum_{n=1}^{2^{k}+1}\left(1-\delta_{k, n}\right) e_{n}$ with $\delta_{k, n}$ the Kronecker symbol, and see that

$$
\sup _{k \geq 1} \frac{\left|h_{k}\right|_{\mathcal{H}_{\tau, q}(\mathcal{B})}}{\left\|h_{k}\right\|_{\mathcal{H}}}=\sup _{k \geq 1}\left(2^{1 / \tau-1 / 2}\right)^{k}=\infty
$$

for any $0<\tau<2$ and $q \in(0, \infty]$.

Now we need to define the sequence $\left\{\varepsilon_{k}\right\}_{k \geq 1}$. Notice that $\mathcal{B}^{\prime}$ is a Riesz basis for $\mathcal{H}$ if, and only if, the operator $T: \mathcal{H} \rightarrow \mathcal{H}$ defined by $T e_{k}=f_{k}$ is bounded and boundedly invertible. For $T$ to be bounded and boundedly invertible, it suffices to have $\|\mid \operatorname{Id}-T\|_{\mathcal{H} \rightarrow \mathcal{H}}<1$. We consider the matrix representation $M$ of the operator $\operatorname{Id}-T$ in $\mathcal{B}$. To ensure that $\||M|\| \|_{\ell_{2} \rightarrow \ell_{2}}<1$, it is sufficient to impose that $\||M|\|_{\ell_{1} \rightarrow \ell_{1}}<1$ and $\||M|\|_{\ell_{\infty} \rightarrow \ell_{\infty}}<1$ (Schur's test), which corresponds to the sufficient conditions

$$
\begin{array}{r}
2^{k}\left|\varepsilon_{k}\right|=\sum_{n=1}^{2^{k}+1}\left(1-\delta_{k, n}\right) \cdot\left|\varepsilon_{k}\right| \leq \delta, \quad \forall k \geq 1 \\
\sum_{k: 2^{k}+1 \geq n}\left(1-\delta_{k, n}\right) \cdot\left|\varepsilon_{k}\right| \leq \delta, \quad \forall n \geq 1
\end{array}
$$

with $0<\delta<1$. Keeping in mind that we also want the resulting Riesz basis $\mathcal{B}^{\prime}$ to be exponentially localized w.r.t. $\mathcal{B}$, we define $\varepsilon_{k}:=2^{-\left(2^{k}+1\right)} \leq 2^{-(k+1)}$ for $k \geq 1$. We notice that $(2.1)$ is obviously satisfied with $\delta:=1 / 2$. Since

$$
\sum_{k: 2^{k}+1 \geq n}\left(1-\delta_{k, n}\right) \cdot\left|\varepsilon_{k}\right| \leq \sum_{k \geq 1}\left|\varepsilon_{k}\right| \leq \frac{1}{2},
$$

both $(2.1)$ and (2.2) are satisfied with $\delta=1 / 2$, and $\mathcal{B}^{\prime}$ is indeed a Riesz Basis for $\mathcal{H}$.

To see that $\mathcal{B}^{\prime}$ is exponentially localized w.r.t. $\mathcal{B}$ with $\lambda=\log 2$ and $C=1$ (see Eq. (1.3)), we let $K=\mathcal{N}=\mathbb{N}$ and notice that for $n>2^{k}+1$, we have $\left\langle f_{k}, e_{n}\right\rangle=0$ so (1.3) is satisfied. The case $n=k$ is obvious, and for $n \leq 2^{k}+1, n \neq k$, we have $|k-n| \leq \max (k, n) \leq 2^{k}+1$, so $-|k-n| \geq-\left(1+2^{k}\right)$, and we obtain

$$
\left|\left\langle f_{k}, e_{n}\right\rangle\right|=\varepsilon_{k}=2^{-\left(2^{k}+1\right)} \leq 2^{-|k-n|} .
$$

Notice that, from the exponential localization of $\mathcal{B}^{\prime}$ w.r.t. $\mathcal{B}$ and the fact that it is a Riesz basis, we have $\mathcal{A}_{q}^{\alpha}\left(\mathcal{B}^{\prime}\right)=\mathcal{H}_{\tau, q}\left(\mathcal{B}^{\prime}\right)=\mathcal{H}_{\tau, q}(\mathcal{B})$.

Now, we let $\mathcal{D}=\mathcal{B} \cup \mathcal{B}^{\prime}$. As a union of two Riesz bases, $\mathcal{D}$ is a frame for $\mathcal{H}$, and it is easy to see that it is quasi-normalized. We claim that $\mathcal{D}$ is exponentially localized w.r.t. $\mathcal{B}$. To see this, we order the elements of $\mathcal{D}=\left\{g_{\ell}\right\}_{\ell \in K}, K:=(\mathbb{N}+1) / 2$ as follows: let $g_{k}=e_{k}$ and $g_{k+1 / 2}=f_{k}$, $k \geq 1$. We notice that

$$
\left|\left\langle g_{k}, e_{n}\right\rangle\right|=\delta_{k, n} \quad \text { and } \quad\left|\left\langle g_{k+1 / 2}, e_{n}\right\rangle\right| \leq 2^{-|k-n|} \leq C 2^{-|k+1 / 2-n|},
$$

which implies $\left|\left\langle g_{\ell}, e_{n}\right\rangle\right| \leq C 2^{-|\ell-n|}, \ell \in K, n \in \mathbb{N}$. 
(C) 2004 Springer. This is the author version of an article published in a Springer journal. The original publication is available at springerlink.com with DOI: 10.1007/s00041-004-3072-z

Thus, $\mathcal{D}$ is an exponentially localized frame w.r.t. $\mathcal{B}$ and for $0<\alpha=1 / \tau-1 / 2<\infty, q \in(0, \infty]$ we have

$$
\mathcal{A}_{q}^{\alpha}\left(\mathcal{B}^{\prime}\right)=\mathcal{H}_{\tau, q}\left(\mathcal{B}^{\prime}\right)=\mathcal{A}_{q}^{\alpha}(\mathcal{B})=\mathcal{H}_{\tau, q}(\mathcal{B})=\mathcal{H}_{\tau, q}(\mathcal{D})=\mathcal{K}_{\tau, q}(\mathcal{D}) \hookrightarrow \mathcal{A}_{q}^{\alpha}(\mathcal{D})
$$

However, since we have $h_{k} \in \Sigma_{2}(\mathcal{D})$ and $\sup _{k} \frac{\left|h_{k}\right|_{\mathcal{H}_{\tau, q}(\mathcal{D})}}{\left\|h_{k}\right\|}=\sup _{k} \frac{\left|h_{k}\right|_{\mathcal{H}_{\tau, q}(\mathcal{B})}}{\left\|h_{k}\right\|}=\infty$ for every $0<$ $\tau<2$ and $q \in(0, \infty], \mathcal{D}$ is far from satisfying any Bernstein inequality. Indeed, no Bernstein inequality and no embedding $\mathcal{A}_{q}^{\alpha}(\mathcal{D}) \hookrightarrow \mathcal{H}_{\tau, q}(\mathcal{B})$ is satisfied for any combination of $\alpha>0$, $q \in(0, \infty]$ and $0<\tau<2$. Thus, the new approximation space $\mathcal{A}_{q}^{\alpha}(\mathcal{D})$ is strictly larger than $\mathcal{A}_{q}^{\alpha}(\mathcal{B})=\mathcal{A}_{q}^{\alpha}\left(\mathcal{B}^{\prime}\right)$.

\section{Conclusion}

We have proved that the exponential localization of a frame with respect to some orthonormal basis is not sufficient to get a Bernstein inequality. The proof is based on the construction of a simple example which is the union of two bases $\mathcal{B}$ and $\mathcal{B}^{\prime}$. This also shows that taking the union of two dictionaries which yield twice the same approximation space can provide a new, strictly larger, approximation space. Thus, the use of even simple redundant dictionaries can drastically improve the approximation power compared to approximation with non redundant bases.

As an example, it is straightforward to check from the results of Jia [9, Theorem 4.1] that with $\mathcal{B}_{1}$ and $\mathcal{B}_{2}$ two "nice" wavelet bases based on two (possibly different) multiresolution analyses, the approximation space is a Besov space and $\mathcal{A}_{\tau}^{\alpha}\left(\mathcal{B}_{1} \cup \mathcal{B}_{2}\right)=B_{\tau}^{\alpha}\left(L_{\tau}(\mathbb{R})\right)=\mathcal{A}_{\tau}^{\alpha}\left(\mathcal{B}_{i}\right)$, $i=1,2, \alpha=1 / \tau-1 / 2$. However, if we build $\mathcal{B}_{1}^{\prime}$ from $\mathcal{B}_{1}$ using our construction, we obtain $\mathcal{A}_{\tau}^{\alpha}\left(\mathcal{B}_{1} \cup \mathcal{B}_{1}^{\prime}\right) \supsetneq \mathcal{A}_{\tau}^{\alpha}\left(\mathcal{B}_{1}\right)=\mathcal{A}_{\tau}^{\alpha}\left(\mathcal{B}_{1}^{\prime}\right)=B_{\tau}^{\alpha}\left(L_{\tau}(\mathbb{R})\right)$. This shows how fragile Bernstein inequalities are with respect to the fine structure of a dictionary, and leaves few hopes of getting any sort of general Bernstein inequality for redundant dictionaries.

\section{ACKNOWLEDGMENTS}

The authors want to thank K. Gröchenig for fruitful discussions leading to the present paper.

\section{REFERENCES}

[1] C. Bennett and R. Sharpley. Interpolation of operators. Academic Press Inc., Boston, MA, 1988.

[2] O. Christensen. An introduction to frames and Riesz bases. Birkhauser, Boston, MA, 2003.

[3] R. A. DeVore and G. G. Lorentz. Constructive approximation. Springer-Verlag, Berlin, 1993.

[4] R. A. DeVore and V. N. Temlyakov. Some remarks on greedy algorithms. Adv. Comput. Math., 5(2-3):173-187, 1996.

[5] R. Gribonval and M. Nielsen. Some remarks on nonlinear approximation with Schauder bases. East J. Approx., $7(3): 267-285,2001$.

[6] R. Gribonval and M. Nielsen. Nonlinear approximation with dictionaries. I. Direct estimates. J. Fourier Anal. and Appl., 2003. to appear.

[7] K. Gröchenig. Localization of frames, Banach frames, and the invertibility of the frame operator. J. Fourier Anal. Appl., 2003. to appear.

[8] K. Gröchenig. Personal communication. December 2002.

[9] R. Q. Jia. A Bernstein-type inequality associated with wavelet decomposition. Constr. Approx., 9(2-3):299318, 1993.

[10] S. B. Stechkin. On absolute convergence of orthogonal series. Dok. Akad. Nauk SSSR, 102:37-40, 1955.

IRISA-INRIA, Campus de Beaulieu, F-35042 Rennes Cedex, France

E-mail address: remi.gribonval@inria.fr

Department of Mathematical Sciences, Aalborg University, Fr. Bajers Vej 7G, DK-9220 AalBorg EASt, Denmark

E-mail address: mnielsen@math.auc.dk 